\title{
Are there any Pharmaceutical Agents Available for the Improvement of Female Sexual Dysfunction (FSD)?
}

\author{
Ana Dias-Amaral*, Diogo Barbosa, Berta Ramos, Vítor Covelo and \\ Márcia Mota \\ Clinical Sexology Outpatient Clinic, Psychiatry and Mental Health Clinic, Centro \\ Hospitalar e Universitário São João, Porto, Portugal
}

*Corresponding author: Ana Dias-Amaral, Clinical Sexology Outpatient Clinic, Psychiatry and Mental Health Clinic, Centro Hospitalar e Universitário São ana.s.d.amaral@gmail.com

\section{Editorial}

Women may not follow a linear model of sexual response (as previously defined by Masters and Johnson). In fact, women seem to respond to sexual cues in a more complex way than men. In her circular model, Basson introduced a new concept of emotional intimacy and emphasized the influence of biopsychosocial factors in women's sexuality. Although the contribution of spontaneous sexual desire in this model is not overlooked, Basson stresses emotional and physical intimacy derived from sexual stimuli and its reinforcing effect on sexual desire and arousal and ultimately in the couple's wellbeing. Thus, Basson's circular model illustrates the multifactorial mature of female sexual response allowing a more comprehensive and effective approach to female sexual dysfunction (FSD).

\section{Framework of Sexual Complaints}

Female sexual complaints are common and the prevalence of FSD is about 30\% [1]. Throughout the last decades, there have been many changes in the definition and categorization of FSD but one thing remains: low sexual desire is the most prevalent dysfunction among women [1]. Although in DSM-V there is no distinction between sexual interest disorder and arousal disorder, most clinical trials still use the previous classification (DSM-IV-TR) which classifies FSD into four categories: sexual desire disorders, sexual arousal disorders, orgasmic disorders and pain disorders. However, these four main categories are not exclusive, and women often complain of more than one difficulty. This poses a demanding challenge to the clinician/therapist when it comes to treat sexual dysfunctions or problems.

\section{Treatment: General Considerations}

There are various treatment options which can include: education, psychotherapy, pelvic floor physiotherapy, alternative treatment elements (such as lubricants, erotica, vibrators, Eros clitoral therapy device), surgery (in selected cases of vulvodynia where vestibulectomy is indicated) and pharmacological treatments. Health care professionals should be aware that not all female sexual complaints are psychological and there are possible pharmacological options, although they still have scarce practical implementation. However, treatment should always be tailored to each woman's particular needs, following the principles of a multidisciplinary and biopsychosocial approach.

\section{Sexual Interest/Arousal Disorders}

When it comes to sexual interest/arousal disorders, several pharmacological agents have been investigated as possible treatment options, and many are used off-label. Testosterone is one of the most commonly studied pharmacological interventions in desire but has not been approved by the Food and Drug Administration (FDA) for 


\section{Mental Health \& Human Resilience International Journal}

the treatment of hipoactive sexual desire disorder (HSDD), being commonly prescribed off-label. The use of testosterone demands caution as long-term risks include breast cancer, insulin resistance and metabolic syndrome. Testosterone supplementation requires concomitant use of estrogens to prevent high testosterone/estrogen ratio which can be an important cardiovascular risk factor. Transdermal and topical formulations are preferable due to low hepatic metabolization. The use of transdermal testosterone patches (in a daily dose of $300 \mathrm{mcg}$ ) in postmenopausal women with HSDD has had positive results with benefits in desire, responsiveness, orgasm and satisfaction, with good safety profile [2-5]. These clinical trials were specifically designed for women who are receiving concomitant oestrogen therapy and for oestrogen-replete surgically and naturally postmenopausal women [6,7]. Therefore, transdermal testosterone patches $(300 \mathrm{mcg} /$ day $)$ are approved by the European Medicines Agency (EMA) since 2006 for the treatment of low sexual desire after surgical menopause in women receiving simultaneous oestrogen therapy. In the future, a recommendation for the use of testosterone in low sexual desire in the context of natural menopause or without oestrogen association is a possibility. Local and systemic oestrogen therapy has also been submitted to clinical trials but official recommendation is lacking in desire disorders [8]. Recent reviews on oestrogen replacement in postmenopausal women have shown benefits on sexual function [9]. Tibolone, an androgenic, progestagenic and estrogenic synthetic hormone, has been used for the treatment of menopausal symptoms, with some evidence showing sexual desire and arousal improvement [10-12]. However, it has not been approved for FSD treatment. Dehydroepiandrosterone (DHEA), the so-called "youth hormone" commonly acquired as a nutritional supplement, has shown positive effects on sexual function in some studies, but the evidence is scarce and even contradictory, therefore, it has not been approved for the treatment of FSD [13,14]. Vasoactive drugs such as sildenafil or alprostadil were submitted to trials on the treatment of FSD but results are inconsistent and approval for the generalized use in clinical practice is lacking. However, sildenafil has shown some positive results in selective serotonin-reuptake inhibitors (SSRI) antidepressants-induced FSD in premenopausal women, in diabetes and multiple sclerosis [15-18]. Bupropion a dopaminergic antidepressant, has shown efficacy either in nondepressed women with HSDD and in women with SSRI-associated sexual dysfunction when used concomitantly $[19,20]$. In this case, bupropion has been associated with a significant increase in self-reported feelings of sexual desire and sexual activity. In recent years, another psychotropic agent has been increasingly used: flibanserin, a $5-\mathrm{HT}_{1 \mathrm{~A}}$ agonist and $5-\mathrm{HT}_{2 \mathrm{~A}}$ antagonist, has received FDA approval for low sexual desire treatment in premenopausal women. However, its clinical efficacy is not completely established as the results seem to vary among different patients [21,22]. Altogether, increase in sexual desire and increase in the number of satisfying sexual encounters seems to be modest $[23,24]$. Bremelanotide a melanocortin receptor agonist has been recently approved by the FDA for HSDD treatment with an efficacy superior to placebo $[25,26]$.

\section{Pain/Penetration Disorders}

Several pharmacological modalities have been used to treat genital pain and penetration disorders. Topical medications have been used alone or in combination with systemic drugs [27]. Topical agents include lidocaine, estradiol, nitroglycerin and amitriptyline or anticonvulsants in topical solutions. Antidepressants as amitriptyline and other tricyclic antidepressants, duloxetine or venlafaxine, and anticonvulsants as gabapentin, pregabalin, topiramate or lamotrigine have been used orally. Some patients have benefited from injectable administration of lidocaine. The majority of treatment options for genital pain and penetration disorders are not evidence-based and have limited success [28]. Some patients experience symptomatic relief but not complete resolution of their complaints.

\section{Orgasmic Disorders}

For the treatment of female orgasmic disorder (FOD) there are no FDA approved drugs. In cases of secondary FOD, the underlying cause should be targeted, sometimes by a specific pharmacological intervention. In most cases, there are no pharmacological options targeting orgasm disorders. Some argue that the pharmacological interventions for the treatment of other sexual disorders (such as desire and arousal disorders) can be relevant in FOD amelioration. Bupropion has already been tested for the treatment of FOD [29].

\section{Final Considerations}

Women's sexual complaints have a complex and multifactorial etiology that poses a demanding challenge in approach and treatment. Therefore, FSD amelioration requires evaluation from different specialists: gynaecologists, psychiatrists, psychologists, endocrinologists, urologists and physical therapists especially trained in sexual medicine. Some 


\section{Mental Health \& Human Resilience International Journal}

pharmacological options are available for FSD treatment and might be useful in selected patients. Scientific evidence exists for the pharmacological treatment of low sexual desire in women after surgical menopause (transdermal testosterone associated with oestrogen replacement) and for SSRI-induced FSD (association with buproprion). The absence of established pharmacological options for FSD treatment arises as an opportunity to discuss non-pharmacological options with patients and to promote further basic and clinical research in this field.

\section{References}

1. Laumann EO, Paik A, Rosen RC (1999) Sexual dysfunction in the United States: prevalence and predictors. JAMA 281(6): 537-544.

2. Buster JE, Kingsberg SA, Aguirre O, Brown C, Breaux JG, et al. (2005) Testosterone patch for low sexual desire in surgically menopausal women: a randomized trial. Obstet Gynecol May 105(5): 944952.

3. Shifren JL, Davis SR, Moreau M, Waldbaum A, Bouchard C, et al. (2006) Testosterone patch for the treatment of hypoactive sexual desire disorder in naturally menopausal women: results from the INTIMATE NM1 Study. Menopause 13(5): 770-779.

4. Simon J, Braunstein G, Nachtigall L, Utian W, Katz M, et al. (2005) Testosterone patch increases sexual activity and desire in surgically menopausal women with hypoactive sexual desire disorder. J Clin Endocrinol Metab 90(9): 5226-5233.

5. Kingsberg S, Shifren J, Wekselman K, Rodenberg C, Koochaki P, et al. (2007) Evaluation of the clinical relevance of benefits associated with transdermal testosterone treatment in postmenopausal women with hypoactive sexual desire disorder. J Sex Med 4(4): 1001-1008.

6. Braunstein GD, Sundwall DA, Katz M, Shifren JL, Buster JE, et al. (2005) Safety and efficacy of a testosterone patch for the treatment of hypoactive sexual desire disorder in surgically menopausal women: a randomized, placebo-controlled trial. Arch Intern Med 165(14): 1582-1589.

7. Kingsberg S (2007) Testosterone treatment for hypoactive sexual desire disorder in postmenopausal women. J Sex Med 4(S3): 227-234.
8. Suckling J, Lethaby A, Kennedy R (2006) Local oestrogen for vaginal atrophy in postmenopausal women. Cochrane Database Syst Rev 8: CD001500.

9. Nappi RE, Polatti F (2009) The use of estrogen therapy in women's sexual functioning (CME). J Sex Med 6(3): 603-616.

10. Wu MH, Pan HA, Wang ST, Hsu CC, Chang FM, et al. (2001) Quality of life and sexuality changes in postmenopausal women receiving tibolone therapy. Climacteric 4(4): 314-319.

11. Laan E, van Lunsen RH, Everaerd W (2001) The effects of tibolone on vaginal blood flow, sexual desire and arousability in postmenopausal women. Climacteric 4(1): 28-41.

12. Uygur D, Yesildaglar N, Erkaya S (2005) Effect on sexual life--a comparison between tibolone and continuous combined conjugated equine estrogens and medroxyprogesterone acetate. Gynecol Endocrinol 20(4): 209-212.

13. Panjari M, Davis SR (2007) DHEA therapy for women: effect on sexual function and wellbeing. Hum Reprod 13(3): 239-248.

14. Panjari M, Bell RJ, Jane F, Wolfe R, Adams J, et al. (2009) A randomized trial of oral DHEA treatment for sexual function, well-being, and menopausal symptoms in postmenopausal women with low libido. J Sex Med 6(9): 2579-2590.

15. Nurnberg HG, Hensley PL, Heiman JR, Croft HA, Debattista C, et al. (2008) Sildenafil treatment of women with antidepressant-associated sexual dysfunction: a randomized controlled trial. JAMA 300(4): 395-404.

16. Caruso S, Rugolo S, Agnello C, Intelisano G, Di Mari L, et al. (2006) Sildenafil improves sexual functioning in premenopausal women with type 1 diabetes who are affected by sexual arousal disorder: a double-blind, crossover, placebo-controlled pilot study. Fertil Steril 85(5): 1496-1501.

17. Caruso S, Rugolo S, Mirabella D, Intelisano G, Di Mari $\mathrm{L}$, et al. (2006) Changes in clitoral blood flow in premenopausal women affected by type 1 diabetes after single 100-mg administration of sildenafil. Urology 68(1): 161-165. 


\section{Mental Health \& Human Resilience International Journal}

18. Dasgupta R, Wiseman OJ, Kanabar G, Fowler CJ, Mikol D (2004) Efficacy of sildenafil in the treatment of female sexual dysfunction due to multiple sclerosis. J Urol 171(3): 1189-1193.

19. Segraves RT, Croft H, Kavoussi R, Ascher JA, Batey SR, et al. (2001) Bupropion sustained release (SR) for the treatment of hypoactive sexual desire disorder (HSDD) in nondepressed women. J Sex Marital Ther 27(3): 303-316.

20. Safarinejad MR (2011) Reversal of SSRI-induced female sexual dysfunction by adjunctive bupropion in menstruating women: a double-blind, placebocontrolled and randomized study. J Psychopharmacol Mar 25(3): 370-378.

21. Anderson R, Moffatt CE (2018) Ignorance Is Not Bliss: If We Don't Understand Hypoactive Sexual Desire Disorder, How Can Flibanserin Treat It? Commentary. J Sex Med 15(3): 273-283.

22. Jaspers L, Feys F, Bramer WM, Franco OH, Leusink P, et al. (2016) Efficacy and Safety of Flibanserin for the Treatment of Hypoactive Sexual Desire Disorder in Women: A Systematic Review and Meta-analysis. JAMA Intern Med 176(4): 453-462.

23. Simon JA, Thorp J, Millheiser L (2019) Flibanserin for Premenopausal Hypoactive Sexual Desire Disorder:
Pooled Analysis of Clinical Trials. J Womens Health (Larchmt) 28(6): 769-777.

24. Clements JN, Thompson B (2018) Flibanserin for hypoactive sexual desire disorder in premenopausal women. JAAPA 31(6): 51-53.

25. Dhillon S, Keam SJ (2019) Bremelanotide: First Approval. Drugs.

26. Althof S, Derogatis LR, Greenberg S, Clayton AH, Jordan R, et al. (2019) Responder Analyses from a Phase $2 b$ Dose-Ranging Study of Bremelanotide. J Sex Med 16(8): 1226-1235.

27. Dias-Amaral A, Marques-Pinto A (2018) Female Genito-Pelvic Pain/Penetration Disorder: Review of the Related Factors and Overall Approach. Rev Bras Ginecol Obstet 40(12): 787-793.

28. Vieira-Baptista P, Donders G, Margesson L, Edwards L, Haefner HK, et al. (2018) Diagnosis and management of vulvodynia in postmenopausal women. Maturitas 108: 84-94.

29. Segraves RT, Clayton A, Croft H, Wolf A, Warnock J (2004) Bupropion sustained release for the treatment of hypoactive sexual desire disorder in premenopausal women. J Clin Psychopharmacol 24(3): 339-342. 